\title{
LA QUESTION DE L'INTELLECT AGENT DANS LE CLIPEUS THOMISTARUM (1481) DE PIERRE SCHWARZ
}

\author{
Serge-Thomas Bonino, O. P. \\ Toulouse
}

\begin{abstract}
RESUMEN
Hacia el final de la Edad Media el fraile dominico Pedro Schwarz (Petrus Nigri) escribió una obra titulada El escudo de los tomistas (Clipeus thomistarum). Es una especie de manual de filosofía que incluye las grandes tesis de la escuela tomista, entre ellas la del entendimiento agente.
\end{abstract}

Palabras clave: Pedro Schwarz (Petrus Nigri), entendimiento agente, Edad Media, Clipeus thomistarum.

\begin{abstract}
At the end of the Middle Ages, the friar Dominican Peter Schwarz (Petrus Nigri) wrote The shield of the Thomists (Clipeus thomistarum). It is a handly book of philosophy in which he presents the main thesis of thomist school, between them is the theory of active intellect.
\end{abstract}

Key words: Peter Schwarz, Active Intellect, Middle Ages, Clipeus thomistarum.

Où en est la question de l'intellect agent dans la scolastique de l'extrême fin du Moyen Âge? Le Bouclier des thomistes (Clipeus thomistarum) du dominicain Pierre Schwarz (Petrus Nigri), ouvrage paru à Venise en 1481 sous les presses de maître Raynald de Novimagio, theutonicus, peu de temps avant la mort de son auteur, constitue un précieux document historique pour répondre à cette question. En effet, le Clipeus thomistarum est une sorte de manuel de philosophie qui présente les grandes thèses de l'école dite thomiste, sur l'horizon d'une confrontation constante avec les autres écoles philosophiques du XV' siècle.

Son auteur, Pierre Schwarz, est né vers 1435 en Bohème'. Il est entré en 1452 chez les frères de l'ordre des prêcheurs, ainsi d'ailleurs que trois de ses frères selon la chair, George, Jean et Nicolas ${ }^{2}$. À l'image de l'ordre dominicain, la formation que Pierre Schwarz reçoit est cos-

1 Sur Pierre Schwarz, cf. S. HeseK, Philosophia Petri Nigri o.p., Dissertatio pro obtinendo doctoratus gradu in facultate philosophica universitatis Friburgensis Helvetorum, 1920; Th. KÄPPELI, Scriptores Ordinis Praedicatorum Medii Aevi, vol. III, Rome, 1980, p. 238-240; B. K. VollmanN, «Nigri (Schwarz), Petrus», Die deutsche Literatur des Mittelalters. Verfasserlexicon, 2. Auflage, Bd. 6, Berlin, 1984, p. 1008-1013.

2 George Schwarz (Georgius Nigri) a lui aussi laissé un nom dans l'histoire dominicaine. Il a accompli ses premières études à Leipzig puis à Bologne. À partir de 1461, il enseigne dans différents studia allemands (Eichstätt, Cologne, Ulm, Regensburg, Ingolstadt...), tout en assurant de nombreuses prédications et en travaillant à la réforme religieuse de son ordre, spécialement comme provincial de Bohème. Il meurt après 1484. George Schwarz a légué une importante collection de sept manuscrits, actuellement conservée à la bibliothèque de l'université d'Eichstätt (Cod. st 683-689). Cf. Maarten J. F. M. HoENEN, Speculum philosophiae medii aevi. Die Handschriftensammlung 
mopolite, largement européenne, puisqu'il étudie à Leipzig (1457) puis à Bologne, grand centre du renouveau thomiste italien du $X V^{e}$ siècle, où un document atteste qu'il est ordonné diacre en 1458. On le trouve ensuite à Montpellier, à Salamanque, où il acquiert une bonne connaissance de l'hébreu, à Fribourg en Brisgau (1471) et à Ingolstat (1473-74). En 1474-75, il dispute contre les juifs à Ratisbonne. Pierre est en effet un des meilleurs hébraïsants de son temps ${ }^{3}$. À ce titre, on lui doit, outre un Tractatus contra perfidos Iudeos de conditionibus veri Messiae, qui est le fruit des controverses de Ratisbonne, un célèbre ouvrage intitulé Der Stern Meschiah, édité dès 1477 à Esslingen. L'œuvre exégétique de notre auteur comprend aussi un Super Psalmos, dédié à Sixte IV (1471-1484). Au plan philosophique, Pierre Schwarz a composé des Quaestiones super libros De anima (1467) ${ }^{4}$ et des Quaestiones super libros Physicorum (1468), les unes et les autres inédites et n'existant que dans un seul manuscrit autographe, signe de leur faible diffusion et probablement de leur faible intérêt. D'une tout autre importance est le Clipeus thomistarum, le bouclier des thomistes (ou encore Pugium thomistarum). Cet ouvrage est dédié à Matthias Corvin ( $\dagger 1490)$, «roi invaincu» de Hongrie et de Bohème. Pierre Schwarz est en effet en 1481 recteur de la jeune université de Buda, où il mourra probablement en 1483. Très vite imprimé et assez largement diffusé, le Clipeus a valu à son auteur une place honorable dans l'école thomiste. Ainsi, lorsqu'en 1621, le P. Côme Morelles publie à Cologne les Questiones super XII libros Metaphisice de Dominique de Flandre, l'édition est ornée d'un frontispice qui représente, réunie symboliquement dans une aula universitaire, autour de saint Thomas enseignant, l'école thomiste: les théologiens d'un côté, les philosophes de 1'autre. Parmi ceux-ci, Petrus niger clipeus thomistarum figure en bonne place entre Armand de Belvézer et Paul Barbo Soncinas. De même, au XVII siècle, le grand théologien thomiste de la province de stricte observance de Toulouse, Jean-Baptiste Gonet, donne à son ouvrage majeur le titre de Clypeus theologiae thomisticae en référence au titre de Pierre Schwarz $z^{5}$. Il reste que l'influence proprement doctrinale du Clipeus de Pierre Schwarz dans le thomisme postérieur est à la mesure de sa qualité: très faible.

Dans son Prologue, Pierre Schwarz dénonce comme un fléau majeur la diversité des opinions philosophiques. Pour remédier à cette confusion des langues, il propose de s'en tenir à un unique auteur: saint Thomas ${ }^{6}$. Il entreprend en conséquence de réfuter les critiques adressées à saint Thomas par les scolastiques postérieurs ${ }^{7}$. C'était déjà, un demi-siècle plus tôt, le projet des Defensiones theologiae divi Thomae de Jean Cabrol ( $\dagger$ 1444), dont le Clipeus est

des Dominikaners Georg Schwartz ( $†$ nach 1484), «Bochumer Studien zur Philosophie, 22», Amsterdam/Philadelphia, 1994.

3 Par exemple, au cour du débat sur l'être et l'essence, Clipeus, I, q. 32, quand il évoque le texte célèbre d'Ex 3, 14, Pierre Schwarz précise: «Esse competit primo agenti per naturam. Esse enim eius est substantia [...] Minor probatur ex. iii 'Sum qui sum. Hoc dices filiis Israel. Esse misit me ad vos'. In hebraice sic habetur Ehied selachani alechem.»

4 Je doute que ce manuscrit apporte des éléments vraiment nouveaux par rapport à la noétique de Pierre Schwarz telle qu'exposée dans le Clipeus, mais une vérification serait utile.

5 Clypeus theologiae thomisticae, authore R.P. Joanne Baptista Gonet..., tomus primus, editio nova, Anvers, 1739, dans la Préface de l'auteur: «Cum impeteretur olim Philosophia Thomistica, suum habuit quo tegeretur Clypeum; aequum est, ut suum habeat quo se tegat, Thomistica Theologia.»

6 Prologue: «Quam itaque ob causam et in sacra theologia et in rerum naturalium humanorumque actuum disciplina unius beati Thome Aquinatis admirabilem celicamque doctrinam conplectendam ab omnibus esse censerem.»

7 Ibid:: «Afferam deinde nonnullos litterarum fama atque scientie prestantissimos viros et quidem ex sacro ordine fratrum minorum Ioannem Scotum quem doctorem subtilem vocant, Franciscum Mayronem quem illuminatum, Petrum Aureoli argumentatorem accerimum, Petrum Aquilanum, Guilielmum Ocham, Antonium Andree atque Landulfum, ex ordine autem hermitarum Gregorium Ariminensem, ex canonicis regularibus Ioannem Cathalanum, atque ex alio genere alios, paulistas, terministas ac nominales quorum omnium in Thomam opiniones argumenta contrarietates destruere, solvere, confutare est hoc in opere propositi mei. Eam quam ob rem hunc ipsum librum quem ad Mathiam Ungarie Boemie gloriosissimum regem inscribo, clipeus thomistarum appello.» 
comme une pâle imitation dans l'ordre purement philosophique. Mais, plus que Jean Cabrol, qui s'en tient fermement au texte thomasien, Pierre Schwarz entend défendre et illustrer saint Thomas en recourant aux auteurs thomistes postérieurs. La liste qu'il dresse à l'occasion des auteurs de l'école thomiste trahit toutefois une redoutable confusion entre institution et doctrine puisque Pierre Schwarz annexe au thomisme tout auteur dominicain ${ }^{8}$. De fait, Pierre Schwarz, comme nous le verrons pour la question de l'intellect agent, n'a qu'une médiocre connaissance du corpus thomasien mais fréquente assidûment des auteurs thomistes postérieurs comme Hervé de Nédellec ou Jean Cabrol. Une étude plus approfondie des sources montrerait sans doute que son originalité est sinon inexistante du moins très réduite?

Comme l'indique son auteur, le Clipeus thomistarum prend la forme d'un commentaire par mode de quaestiones de l'ars vetus d'Aristote, genre littéraire alors très commun dans l'enseignement universitaire ${ }^{10}$. Le style en est on ne peut plus scolaire. Pierre recourt aux procédés usuels de la pédagogie scolastique. Il dresse des status questionis présentant les diverses opinions en présence sur un point déterminé, énonce des thèses qu'il justifie par des démonstrations syllogistiques, soulève des dubia..., tout cela dans un style sans aucun ornement littéraire ${ }^{11}$. Après un certain nombre de questions préliminaires sur l'objet et le statut de la logique, le livre I est consacré à l'explication de l'Isagoge de Porphyre qui est une introduction aux Catégories. Le livre II commente les Catégories elles-mêmes ainsi que le De sex principiis de Gilbert de la Porrée que les scolastiques considéraient comme un complément aux Catégories. Mais Pierre Schwarz donne à son «commentaire» une ampleur considérable puisqu'il y touche à nombre de questions qui dépassent l'objet précis de l'ars vetus. Ainsi des problèmes de physique, de théologie naturelle ou encore de psychologie rationnelle y côtoient les questions de stricte logique... C'est ainsi que Pierre consacre plusieurs questions à la doctrine de l'intellect agent.

En effet, à chacune des dix catégories discernées par Aristote, Pierre Schwarz rattache, plus ou moins artificiellement, toutes sortes de questions qui renvoient aux principaux «débats» philosophiques de son temps. La question de l'intellect agent prend place à l'intérieur des développements sur la catégorie de la qualité (q. 44-60). Au milieu de questions concernant directement le statut de la qualité, ses «espèces», ses propriétés remarquables (avec, en particulier, la question de l'intensification des formes [q. 56-58]), un ensemble de dix questions (q. 46-55) traite en fait de psychologie rationnelle et de noétique. Le rattachement à la catégorie de la qualité se justifie dans la mesure où, dès la q. 46, qui ouvre la série, Pierre Schwarz établit, contre Durand de Saint-Pourçain, que l'intellection est une qualité positive et non pas une simple relation entre le sujet et l'objet.

8 Ibid:: «Postremo vero subiiciam que ad ipsius Thome sententias ex aliis auctoribus adiicienda esse delegerim ut opus habeam copiosum pariter atque perfectum. Sectatus sum quidem ex ordine predicatorum celebratissimos viros Albertum colonensem quem vulgo magnum appellatur, Herveum britonem acutissimum, Petrum de Palude, Guilielmum de Maricalmo, Ioannem Capreoli, tholosanum, ac loannem neapolitanum, ceterosque professionis eiusdem doctores illustres quibus in sancti doctoris libris [?] atque doctrina plurimum luminis enanscici potest.» En I, q. 22, à propos des universaux, Pierre Schwarz cite comme thomistes des auteurs pour le moins innatendus, dont le seul point commun est en fait d'être dominicains: «De hoc est opinio omnium thomistarum, scilicet Herphei, Durandi, Petri de Palude, Thome anglici Holkot, Bernardi Lombardi, Guilielmi de Maricalmo. Et Capreoli.»

9 Comme on le verra, j'ai pu établir sans trop de peine la dépendance littéraire du Clipeus par rapport à Hervé de Nédellec, Jean Cabrol ou Jean Duns Scot. Mais Pierre Schwarz avait aussi accès à de nombreux auteurs secondaires de la tradition thomiste et de la tradition scotiste (comme François de Mayrones, Antoine Andrée...), qu'il cite parfois et dont il est probable qu'il aussi pille les textes.

10 Prologue: «Sequar hunc ordinem. Inprimis enim ad modum commenti super Aristotelis dyalectica veteri questiones inducam ad liberales artes atque philosophiam plurimum conducentes.»

11 Prologue: «Stilo itaque utar minime gravi vel oratorio sed admodum plano atque scolastico et ad philosophiam atque dyalecticam accomodato.»

12 Q. 46: Utrum actus intelligendi sit quid absolutum vel respectivum formaliter. 
Pierre Schwarz n'a manifestement pas suivi la recommandation méthodologique de l'Aquinate dans le Prologue général de la Summa theologiae qui veut que le développement de l'exposé, l'ordo disciplinae, se prenne de l'objet lui-même. En effet, l'ordre de ces dix questions est un ordre plutôt matériel qui dépend surtout de l'ordre des textes des auteurs que Pierre suit ou combat. Ces questions de psychologie rationnelle sont donc loin de former une synthèse harmonieuse.

Le premier adversaire affronté est Durand de Saint-Pourçain, grand pourfendeur de la noétique aristotélicienne et coryphée des négateurs de l'intellect agent. Contre le dominicain dissident, Pierre Schwarz commence par établir que l'acte d'intelliger n'est pas une simple relation entre le sujet et l'objet mais une réalité positive absolue, en fait une qualité (q. 46) ${ }^{12}$. Pour cela, il s'inspire massivement de la q. 8 du Quodlibet III d'Hervé de Nédellec ( $†$ 1323), qui est, après (?) saint Thomas, l'auteur préféré de Pierre Schwarz. Comme Hervé de Nédellec, Pierre s'interroge ensuite sur les causes de l'intellection, entrant ainsi dans l'analyse du processus même de l'intellection (q. 47) ${ }^{13}$.

La puissance intellective tenant une place déterminante parmi ces causes de l'intellection, Pierre Schwarz amorce alors un développement sur les puissances de l'âme, dont la source directe est ici non plus Hervé de Nédellec mais Jean Cabrol ${ }^{14}$. Contre les scotistes d'une part et contre Guillaume d'Ockham d'autre part, il montre que les puissances de l'âme se distinguent réellement de l'essence de l'âme (q. 48) ${ }^{15}$.

Après ce détour, notre auteur revient, non sans se répéter quelque peu, à la réfutation de la noétique de Durand de Saint-Pourçain et s'attache plus directement au problème de l'intellect agent. Il soulève à son propos deux questions: existe-t-il (q. 49) ${ }^{16}$ ? Quel est son rôle dans le processus de l'intellection (q. 50) ${ }^{17}$ ? Les deux questions sont connexes puisque Durand déduisait la non-existence de l'intellect agent de sa parfaite inutilité dans le processus intellectif.

Revenant ensuite au statut ontologique de l'intellect agent, Pierre Schwarz précise, contre Jean Duns Scot, que l'intellect agent est réellement distinct de l'intellect possible (q. 51$)^{18}$. Duns Scot semble pourtant être la grande source d'inspiration lorsqu'il s'agit, dès la question suivante, de défendre, contre Henri de Gand, la nécessité d'une species intelligible dans le processus de l'intellection (q. 52) ${ }^{19}$ et, par suite, l'existence d'une double mémoire, sensible et intellectuelle (q. 53) ${ }^{20}$. Les deux dernières questions de cet ensemble portent sur les rapports controversés de l'intelligence et de la volonté et ne nous intéressent.pas directement ${ }^{21}$.

Je me propose donc d'analyser cet ensemble de questions en prêtant une attention toute particulière (ce qui s'appelle abstraire!) à l'enseignement relatif à l'intellect agent, puis de tenter quelques conclusions historiques et doctrinales.

13 Q. 47: A quo causatur actus intelligendi.

14 C. Johannis Caprioli Tholosani ordinis praedicatorum thomistarum principis Defensiones theologiae divi Thomae Aquinatis de novo editae cura et studio RR. PP. Ceslai Paban et Thomae Pègues, 7 vol., Tours, 1900-1908, I, d. 3, q. 3 (t. 1, p. 189 ss).

15 Q. 48: Utrum potentie anime distinguantur ab anima.

16 Q. 49: Utrum sit dare intellectum agentem.

17 Q. 50: In quid agat intellectus agens.

18 Q. 51: Utrum intellectus agens et possibilis sint distincte res.

19 Q. 52: Utrum necesse sit ponere in parte intellectiva aliquam speciem intelligibilem preter illam que est in fantasmate.

20 Q. 53: Utrum memoria sit in parte intellectiva vel in parte sensitiva tantum.

21 Q. 54: Utrum intellectus et voluntas sint idem realiter; q. 55: Utrum intellectus sit nobilior voluntate. 


\section{L'INTELLECTION (Q. 46-47)}

La partie noétique du Clipeus s'ouvre donc par deux questions (q. 46-47) qui reprennent sans grande originalité l'enseignement d'Hervé de Nédellec à la q. 8 de son Quodlibet III ${ }^{22}$. En 1309 d'après J. Koch, le dominicain breton y réfutait soigneusement la thèse de son confrère bourbonnais, Durand de Saint-Pourçain, selon laquelle l'acte d'intelliger désignerait formellement non une réalité positive mais un simple rapport $\mathrm{t}^{23}$.

Cette thèse de Durand fait partie d'une offensive radicale contre la noétique aristotélicienne (et donc thomiste), qui a suscité la réaction virulente de ses confrères thomistes ${ }^{24}$. Plus précisément, l'intention de Durand en réduisant l'intellection à une simple mise en relation de l'intellect avec l'objet rendu présent dans l'image était de mettre en relief, dans une perspective augustinisante, la spontanéité de l'esprit dans la connaissance, à l'encontre d'une conception aristotélisante où l'intellect risquait selon lui d'apparaître trop passif face à son objet ${ }^{25}$. Pour Durand, l'intellection n'ajoute donc rien de positif à l'intellect; elle n'est d'aucune manière un acte second, une forme, qui viendrait déterminer et parfaire la puissance intellective ${ }^{26}$. En effet, pour Durand, l'intellect est toujours en acte et n'a donc aucun besoin d'être actualisé. Exit, par conséquent, la species intelligible comme forme intentionnelle censée actualiser et déterminer l'intellect en vue de l'intellection ${ }^{27}$, et, avec elle, la causalité de l'objet extra-mental. Celui-ci, est destitué du statut de cause pour n'être plus qu'une occasion ou cause sine qua non de l'intellection, en tant qu'il est le terme atteint par elle, ce sur quoi elle porte. Exit aussi l'intellect agent qui était censé produire la species et actualiser ainsi (ou d'une autre manière) l'intellect.

22 Hervaeus Natalis, Quodlibeta, III, q. 8 (Venise, 1513, f. 78rb-80 vb). Cette question a aussi été éditée dans Durandi de S. Porciano o.p, Quaestio de natura cognitionis (II Sent. [A] D. 3 Q. 5) et Disputatio cum anonymo quodam nec non Determinatio Hervei Natalis O.P. (Quol. III Q. 8), ad fidem manuscriptorum edidit J. Koch., «Opuscula et textus historiam Ecclesiae eiusque vitam atque doctrinam illustrantia. Series scholastica, $6 »$, Münster i. W, 1929 , p. 43-75.

23 Actus intelligendi dicit formaliter respectum. Durand soutient cette thèse dans la seule première rédaction de son Commentaire, II, d. 3, q. 5 . Le texte en a été édité par J. KocH, op. cit., p. 7-32. Elle constitue la dix-huitième proposition stigmatisée par la censure de juillet 1314: «D. 3 a. 5 in suo veteri dicit quod intelligere, sentire et omnis operacio manens in agente non dicit aliquid realiter differens ab intellectu, sensu et huiusmodi, faciens cum eis composicionem; et quod intellectus non est perfeccior, cum actu intelligit quam ante intelligere per se, sed solum per accidens sicut grave cum est deorsum; nec plus differunt talia quam albedo et albere, calor et calere, quia unum designat nominaliter, aliud autem verbaliter'. Nec iste eciam articulus est in suo novo. Omnia sunt falsa et erronea et [contra] doctrinam communem.» (J. KoCH, Kleine Schriften, II, «Storia e letteratura, 128», Rome, 1973, p. 5758), ainsi que la cinquante-huitième proposition censurée pour s'opposer à la doctrine de Thomas (ibid., p. 84-85). Cette thèse caractérise pour les médiévaux la noétique de Durand. Jean Cabrol l'attribue explicitement à Durand en In I Sent., d. 35, q. 1, a. 1 (t. II, p. 355b. Sa source est Pierre Auriol, In I Sent., d. 35, q. 1, a. 1) et la présente, avec les mêmes arguments mais sans mentionner Durand, en In II Sent., d. 3, q. 2 (t. III, p. 258b-259b. Sa source est Grégoire de Rimini, In II Sent., d. 7, q. 2, ed. A. Trapp, V. Marcolino, M. Santo-Novas, t. 5, Berlin-New York, 1979 , p. 85 ss.).

24 Cf. S. Th. Bonino, «Quelques réactions thomistes à la critique de l'intellect agent par Durand de SaintPourçain», dans Saint Thomas au XIV exp. e siècle, Actes du colloque organisé par l'Institut Saint-Thomas d'Aquin de Toulouse, Revue thomiste 97 (1997), p. 99-128.

25 La critique du passivisme aristotêlicien apparaît clairement dans un texte des Additiones cité par J. KoCH, Durandus de Sancto-Porciano o.p. Forschungen zum Streit um Thomas von Aquin zu Beginn des 14. Jahrhunderts. I. Teil: Literargeschichtliche Grundlegung, «Beiträge zur Geschichte der Philosophie des Mittelalters, 26», Münster i. W., 1927, p. 44-45: «Nullum obiectum movet effective potentiam quancumque sensitivam vel intellectivam ad cognitionem sui, sed solum est causa sine qua non. [...] Anima immaterialis unita corpori movet corpus et a corpore non movetur, nec aliquid recipit a corpore.»

26 Si Durand réduit l'intellection à une relation, il importe cependant de noter que la relation est, chez Durand, plus qu'un simple accident. Elle est un véritable mode d'être. Cf., les remarques de G. EMERY, «La théologie trinitaire des Evidentiae contra Durandum de Durandellus», dans Saint Thomas au XIV exp. e siècle..., p. 180-205.

27 Cf. la critique de la species intelligible par Durand en In II Sent., d. 3, q. 6 (In Petri Lombardi Sententias theologicas Commentariorum libri IIII, Venise, 1586, f. 139ra-140rb). 
Le modèle noétique durandien est un modèle minimal: la puissance intellectuelle, à qui revient l'initiative, et l'objet se font face, à l'exclusion de toute autre instance.

Dans la q. 46 du livre II du Clipeus, Pierre Schwarz commence par résumer l'exposé que fait Hervé de Nédellec de la position de Durand ${ }^{28}$. Il présente ensuite la triple critique qu'Hervé adresse à Durand ${ }^{29}$ mais laisse de coté la suite de la réfutation pour en venir à l'énoncé de la thèse d'Hervé à laquelle il se rallie ${ }^{30}$ :

«Dans la puissance intellective, quand l'âme intellige en acte, se produit un acte absolu qui formellement n'est pas une relation, bien qu'il entraine une relation, et l'intellect est dit formellement intelliger en acte par cet acte au titre de sujet (subiective). Mais, au titre de cause efficiente (effective), un tel acte vient de l'intellect agent et de l'objet, ou de la species selon d'autres. ${ }^{31}$ »

La distinction qu'établit Hervé de Nédellec entre la cause subjective et la cause efficiente de l'intellection est déterminante dans sa noétique. Pour Hervé, l'opération d'un principe réceptif ou subjectif (comme l'intellect possible) s'identifie avec la forme reçue ou avec le fait de recevoir cette même forme, tandis que l'opération d'un principe efficient (comme l'intellect agent, l'objet ou la species) est nécessairement distincte de celui-ci. L'intellection étant l'effet de ses causes efficientes, elle est distincte d'elles.

A plusieurs reprises (q. 47, q. 49, q. 5132), Pierre Schwarz renvoie explicitement à ses propres développements du livre I du Clipeus, q. 5, sur cette nécessaire distinction entre la cause efficiente et le sujet. Dans cette importante question, Pierre Schwarz définit les sept propriétés qui d'après lui caractérisent le sujet d'une science:

«Le sujet d'une science est un étant réel présupposé à la totalité de la science et ayant des parties définitives et des propriétés réellement distinctes de lui-même ${ }^{33}$.»

La cinquième propriété, à savoir la distinction entre le sujet lui-même de la science et ses parties, amène un long développement anti-scotiste et anti-nominaliste $\mathrm{Au}$ terme, Pierre aborde quelques dubia, c'est-à-dire quelques problèmes. Le sujet, explique-t-il tout d'abord, est bien cause de la propriété mais il n'en est pas la cause efficiente, car, selon Hervé de Nédellec, la cause efficiente et la cause matérielle ne peuvent jamais coïncider ${ }^{34}$. En effet, il s'agit là d'une

28 Les quatre arguments de Pierre Schwarz correspondent aux quatre arguments exposés par Hervé (Quodlibeta, 78va-b). Mais Pierre Schwarz n'a pas repris l'exposé préliminaire par lequel Hervé entendait donner une certaine intelligence de la position de Durand (78va).

29 Cf. «Contra istam opinionem arguit Herpheus quodlibeto $I I, q .8$, tribus viis.» Cf. petilco capitales Hervé, Quodlibeta, III, q. 8 (78vb-79rb). Pierre Schwarz ajoute, semble-t-il, un argument de son crû: tout habitus est engendré par la répétition d'actes. Or si l'acte d'intelliger est une simple relation, alors que l'habitus est une réalité positive, le moins aura engendré le plus, ce qui est absurde.

30 La fin de la q. 46 reprend sans originalité les réponses d'Hervé aux objections de Durand (79rb-79va).

31 «Dicit igitur Herpheus quod in potentia intellectiva quando anima actu intelligit fit quidam actus absolutus qui formaliter non dicit relationem licet relatio consequitur ad ipsum et intellectus dicitur formaliter intelligens tali actu subiective. Effective autem talis actus est ab intellectu agente et ab obiecto vel a specie secundum alios.» On notera la réserve d'Hervé sur la species intelligible. Cf. L. SPRUIT, Species intelligibilis, From Perception to Knowledge, vol. 1: Classical Roots and Medieval Discussions, «Brill's Studies in intellectual History, 48», Leiden, 1994, p. 270-273.

32 Cf. q. 47; «ut declaratum fuit quinta questione super Porphyrio... in solutione prime Scoti»; q. 49: «Alias probationes eiusdem maioris require questione quinta super Porphirio conditione quinta subiecti scibilis»; q. 51: «Istam rationem habes deductam super Porphirio questione quinta conditione quinta subiecti scibilis dubio secundo et adducitur pro ratione Scoti.»

33 «Subiectum scibile est ens reale toti scientie presuppositum habens partes diffinitivas et passiones realiter a se distinctas.»

34 Pierre renvoie à la d. 3 du livre I des Sentences. Cf. Herveus NATALIS, In quatuor libros Sententiarum Commentaria, I, d. 3, q. 4 (Paris, 1647, p. 38-41). 
application du principe, examiné dans le second dubium, selon lequel une chose ne peut être à la fois en acte et en puissance par rapport à une seule et même chose. Pierre Schwarz signale que cette question, ici soulevée à propos du rapport entre le sujet d'une science et ses propriétés, a néanmoins une valeur principielle, architectonique, puisqu'elle gouverne au moins quatre autres problèmes majeurs:

«L'intellect agent et l'intellect possible sont-ils une seule chose absolue? L'âme qui a des puissances réellement distinctes d'elle peut-elle être dite leur cause efficiente? La volonté peut-elle se mouvoir elle-même? Les corps lourds et les corps légers se meuvent-ils d'eux-mêmes au point d'être causes efficientes du mouvement qui est en eux comme dans leur sujet ${ }^{35}$.»

\section{La réponse de l'école est unanime:}

«La cause efficiente et la matière ne coïncident jamais numériquement, comme le dit le Philosophe au livre II de la Physique, puisque une même chose ne peut être en acte et en puissance par rapport à la même chose ${ }^{36} . \gg$

\section{Aussitôt Pierre Schwarz ajoute:}

"Aussi, comme l'intellect possible reçoit l'intellection, il ne peut causer cette même intellection. C'est la raison pour laquelle on pose un intellect agent qui cause de façon efficiente l'intellection que reçoit l'intellect possible ${ }^{37} . »$

A cette thèse générale s'oppose l'opinio Scoti selon laquelle une seule et même chose peut être en même temps en puissance formelle et en acte virtuel par rapport à une certaine perfection. Elle est surtout destinée à fonder la possibilité d'une auto-motion de la volonté, mais elle justifie aussi aux yeux de Scot le refus d'introduire une distinction réelle entre l'intellect agent et l'intellect possible. Pierre Schwarz réfute longuement cette thèse, puis il tire trois corollaires de cette critique. Le premier est que la volonté ne peut se mouvoir elle-même. Le second contient en germe tout l'enseignement noétique de Pierre Schwarz:

«Il est impossible que l'intellect agent et l'intellect possible soient une seule et même chose. Preuve: l'intellect possible reçoit l'acte d'intelliger. Il ne peut donc le causer sinon une même chose serait en acte et en puissance par rapport à une seule et même chose. Bien plus, il est nécessaire que quelque chose d'absolu, autre que l'intellect possible, soit cause efficiente de l'acte d'intelliger. L'objet n'est pas la cause totale de l'acte d'intelliger, mais seulement cause partielle et moins principale, car lorsque je pense la blancheur, l'acte de penser la blancheur vaut mieux que la blancheur et ne peut donc être causé par

35 «Istud dubium pro tanto est ad propositum utrum videlicet sit possibile quod subiectum sit causa effectiva sue passionis cum sit causa subiectiva. Item utrum intellectus agens et possibilis sunt una res absoluta. Item utrum anima habens potentias realiter a se distinctas possit dici esse causa effectiva earumdem. Item utrum voluntas possit movere seipsam. Item utrum gravia et levia moveantur a seipsis ita quod gravia et levia sint cause effective motus qui in eis est subiective.»

36 «Ad istam questionem respondet communis scola quod efficiens et materia numquam coincidunt in idem numero, ut dicit Philosophus $2^{\circ}$ phisicorum quia idem respectu eiusdem non potest esse in actu et in potentia.» $\mathrm{Cf}$. ARIstote, Physique, II, c. 7 et ThOMAs D'AQUIN, In octo libros de physico auditu sive Physicorum Aristotelis commentaria, II, lect. 11 (Naples, 1953, p. 111, $\mathrm{n}^{\circ} 474$ ): «Materia vero non est nec idem specie nec idem numero cum aliis causis: quia materia inquantum huiusmodi est ens in potentia, agens vero est ens in actu inquantum huiusmodi, forma vero vel finis est actus vel perfectio.»

37 «Unde quia intellectus possibilis recipit ipsam intellectionem ideo non potest causare eandem. Et ea ratione ponitur intellectus agens qui causet effective intellectionem quam intellectus possibilis recipit.» 
la blancheur comme cause principale. Et la blancheur ne peut par sa vertu propre mouvoir l'intellect possible, car tout moteur vaut mieux que ce qu'il meut. Il faut donc que ce qui meut l'intellect et cause l'acte d'intelliger vaille mieux que l'un et l'autre. Ce n'est le cas que de l'intellect agent. L'intellect agent diffère donc réellement de l'intellect possible. On le dira mieux ailleurs ${ }^{38}$."

L'intellect agent apparaît ici comme une des causes efficientes, la principale, de l'intellection dont l'intellect possible est le sujet récepteur. L'accent est donc vigoureusement placé sur la passivité radicale de l'intellect possible, ramené au rôle de réceptacle passif de l'intellection. Pierre Schwarz semble ainsi réduire le processus noétique à une action transitive réelle que l'objet (éventuellement, la species) et l'intellect agent exercent en commun sur l'intellect possible. D'ailleurs, il explique, en s'appuyant sur Hervé de Nédellec, qu'il n'y a pas d'action proprement immanente, que l'action dite immanente est en fait une forme d'action transitive ${ }^{39}$ ! Il exténue ainsi la spontanéité propre de l'esprit, d'une manière qui ne correspond guère à l'enseignement de saint Thomas. Celui-ci, tout en insistant sur la dépendance de la pensée vis-à-vis de l'objet, voyait dans l'intellect possible davantage qu'un simple sujet récepteur: un co-principe de cette activité immanente qu'est l'intellection.

Mais revenons à nos q. 46-47. Hervé de Nédellec avait bien compris que la thèse de Durand sur la nature de l'intellection comme pure relation n'était pas un dérapage anodin mais la partie émergée d'une redoutable noétique générale. Dans la q. 8 de son Quodlibet III, la critique de la nature de l'intellection était donc immédiatement suivie d'un exposé critique de l'analyse durandienne des causalités à l'œuvie dans l'intellection. Pierre Schwarz reprend ce développement d'Hervé pour en faire une question à part entière, la q. 47: «Qu'est-ce qui cause l'acte de penser?».

Deux opinions s'opposent. La première est celle de Durand que Pierre présente en suivant fidèlement l'exposé d'Hervé de Nédellec ${ }^{40}$. Comme celui-ci, il ramène à trois thèses ou conclusions la position de Durand: l'acte d'intelliger n'est pas causé par la species et l'objet; l'intelliger ou acte d'intelliger est causé par l'objet comme par une cause sine qua non; l'acte d'intelliger est causé par celui qui donne la puissance intellective [Dieu] comme par une cause par laquelle il advient. Ces trois thèses définissent bien le spontanéisme de Durand qui tend à affaiblir la causalité de l'objet extra-mental au profit de l'activité propre de l'esprit.

La réfutation des thèses durandiennes incombe ici encore à Hervé de Nédellec ${ }^{41}$. L'apport propre de Pierre Schwarz (outre la sélection simplificatrice qu'il opère dans le texte d'Hervé) est ici, comme souvent, assez maigre. Il se ramène à deux éléments.

Premièrement, pour prouver que la species intelligible n'est pas une cause nécessaire de l'intellection, Durand de Saint-Pourçain faisait valoir que, l'intellection étant un acte immanent qui demeure en ce dont il est l'acte, il faudrait, si la species était principe de l'intellection, qu'intellection et species soient absolument inséparables. Or, il y a au moins un cas, où elles

38 «Impossibile est quod intellectus agens et possibilis sint una res realiter. Probatur. Quia intellectus possibilis suscipit actum intelligendi, igitur eum causare non potest, quia alias respectu eiusdem actus esset in actu et in potentia. Ideo oportet quod aliud ab intellectu possibili absolutum sit causa effectiva actus intelligendi. Non autem obiectum est causa actus intelligendi totalis nisi forsan partialis et minus principalis quia quando intelligo albedinem, tunc actus intelligendi est nobilior albedine. Igitur non potest causari ab albedine tanquam a causa principali. Nec albedo virtute propria postet movere intellectum possibilem, quia omne movens est nobilius motu. Oportet igitur quod illud quod movet intellectum et causat actum intelligendi sit nobilius utroque. Hoc autem non est nisi intellectus agens. Igitur intellectus agens differt realiter ab intellectu possibili, de quo alias melius.»

39 Cf. I, q. 5: «Respondet Herpheus quodlibeto secundo questio octava quod improprie dicitur actio manens. et actio transiens, quia nulla est actio manens in agente ut probatum est, sed debet dici operatio manens operatio transiens, quia operatio est commune quid ad effectus omnium causarum, ut materie operatio est materiale, forme operatio informare, efficientis operatio est effective causare, finis operatio finire.»

40 Cf. Quodlibeta, III, q. 8 (f. 79ra-80ra). 
sont séparées, celui de la vision béatifique. Hervé répondait en distinguant le sujet de l'intellection de sa cause efficiente: l'intellection n'est pas l'opération immanente de la species intelligible ou de quelque autre cause efficiente que ce soit mais de l'intellect possible! Or Pierre Schwarz ne reprend pas ici la réponse d'Hervé, mais emprunte une autre voie pour débouter Durand. Alors qu'Hervé attaquait la consequentia, Pierre s'en prend à l'antecedens. Il conteste la validité du recours à l'ordre surnaturel pour déstabiliser les structures de l'ordre naturel ${ }^{42}$. Il faut, selon lui, distinguer fermement l'ordre naturel —où il n'y a jamais intellection sans species- et l'ordre surnaturel, dont relève la vison béatifique. Toutefois, comme l'ordre surnaturel ne contredit pas la nature, Pierre admet que, dans la vison béatifique, Dieu joue analogiquement le rôle qui est celui de la species dans la connaissance naturelle. Mais ce rare accès d'originalité ne trahit pourtant aucune prise de distance vis-à-vis d'Hervé ${ }^{43}$.

Deuxièmement, le premier argument de Durand contre la causalité de l'objet dans l'intellection inclue une critique de l'intellect agent (sans lequel l'objet ne peut effectivement agir sur l'intellect possible). Or, dans cette critique, Durand fait intervenir un parallélisme supposé entre sens agent et intellect agent. Or les scolastiques, à commencer par saint Thomas ${ }^{44}$, nient généralement la nécessité d'un sens agent, c'est-à-dire d'une faculté sensible dont le rôle serait de rendre l'objet sensible apte à produire une sensation ${ }^{45}$. Pour Durand, il n'y a pas plus besoin d'intellect agent que d'un sens agent. À cette objection, Hervé de Nédellec proposait comme deux solutions ${ }^{46}$. La première tient contre Durand l'existence d'un sens agent et s'efforce en conséquence de justifier le silence du Stagirite sur ce point. Si Aristote, explique Hervé, n'a pas parlé du sens agent, c'est parce que le sens ne transcende pas les qualités sensibles de façon aussi nette et évidente que l'intellect transcende les quiddités matérielles. La seconde solution prend acte de l'inexistence du sens agent. Le décalage ontologique entre l'objet sensible et la faculté sensible est comblé par l'influx extrinsèque d'une vertu spirituelle, c'est-à-dire d'une de ces intelligences séparées qui peuplent et meuvent les sphères célestes. Mais il en va différemment dans l'ordre proprement intellectuel.

Or Pierre Schwarz illustre cette double réponse possible par la référence à deux auteurs thomistes. Le premier est Guillaume de Maricalm. On ne sait absolument rien de ce maître dominicain — non memoratur in historia, dit S. Herek ${ }^{47}$ — sinon ce qu'en dit Pierre Schwarz qui le cite positivement à plusieurs reprises comme dominicain, thomiste et auteur d'un commentaire des Sentences. Or Guillaume est partisan de l'existence d'un sens agent:

«Maître Guillaume de Maricalm tient la même position au livre I des Sentences, d. 3, dans la question: est-ce que l'intellect agent est une partie de l'image qui est la mémoire? Il faut poser le sens agent d'une part à cause de la transcendance de la puissance sensitive sur le sens et d'autre part à cause de la transcendance de l'acte de sentir dont l'objet sensible ne peut être la cause suffisante ${ }^{48},{ }^{\prime \prime}$

41 «Ad ista argumenta respondet Herpheus Quodlibeto III, q. 8.»

42 Nous retrouverons à la q. 51 une affirmation nette de la distinction entre ordre surnaturel et ordre naturel. Mais Pierre n'hésite pas par ailleurs à appuyer ses thèses philosophiques sur des arguments théologiques. Par exemple, à la q. 52, pour établir l'existence đ'une mémoire intellectuelle, il se réfère à la parabole évangélique du pauvre Lazare et du mauvais riche, où il appert que dans l'autre monde les hommes gardent mémoire de leurs actions d'ici-bas.

43 Dès sa réponse à la deuxième objection de Durand, Pierre reprend la cistinction fondamentale d'Hervé entre cause matérielle subjective et cause efficiente.

44 Cf., par exemple, Summa theologiae, I, q. 79, a. 3, ad 1.

45 Sur la problématique du sens agent, cf., par exemple, A. PATTIN, Pour l'histoire du sens agent, La controverse entre Barthélemy de Bruges et Jean de Jandun, Ses antécédents et son évolution, Etude et textes inédits, «Ancient and medieval Philosophy, Series 1, VI», Leuven, 1988.

46 Cf. Quodlibeta, III, q. 8 (f. 80 rb-va).

47 S. HESEK, op. cit., p. 26.

48 «Idem tenet magister Guilielmus de Maricalmo in primo scripto distinctione tertia in illa questione utrum 
Le second thomiste est Pierre de la Palu ( $†$ 1342) qui, au livre IV de son Scriptum, nie l'existence d'un sens agent $t^{49}$ : 1 'objet sensible est capable de produire une species dans la puissance sensitive, soit par lui-même, soit sous l'influx d'une vertu cosmique.

Pierre Schwarz reviendra encore sur cette question du sens agent à la q. 49, toujours pour réfuter Durand. Dans ce dernier texte, il semble pencher personnellement en faveur de l'opinion de Guillaume de Maricalm:

«Il n'est pas nécessaire de poser un sens agent à cause de la présence de l'objet sensible à la puissance sensitive, ni à cause de l'impression de la species qui est selon la même ratio dans le milieu et dans l'organe. Pourtant, il est nécessaire de le poser à cause de la transcendance de la puissance sensible sur le sensible et à cause de la transcendance de l'acte de sentir qui ne peut être causé de façon suffisante par l'objet sensible ${ }^{50} . »$

L'objet sensible se rend présent par lui-même, mais il y a une disproportion causale entre l'objet sensible et la sensation, dont il ne peut être la seule cause.

\section{L'EXISTENCE DE L'INTELLECT AGENT (Q. 49)}

Dans la q. 47, Pierre Schwarz, en exposant la négation durandienne de la causalité de l'objet dans l'intellection, ne pouvait pas ne pas faire référence à sa critique de l'intellect agent. Mais il y revient plus directement dans les qq. 49-50.

Durand de Saint-Pourçain n'est pas nommé dans la q. $49-\ll Y$ a-t-il un intellect agent?»—, mais la première opinion, celle qui conteste la nécessité d'un intellect agent, est manifestement la sienne. Deux arguments durandiens sont retenus. Le premier, comme nous ve-

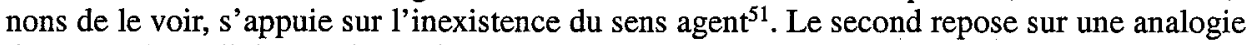
de proportionnalité entre la production d'un effet physique et la production de la pensée. La production d'un effet physique n'exige, selon Durand, que deux instances: un agent universel (le soleil) et une vertu active qui est comme le seminarium virtutis universalis. De même, la production de l'intellection n'exige que deux instances: l'intellect séparé (Dieu) et les objets créés qui comme des semences contiennent les quiddités. Pierre rétorque que cette analogie joue plutôt en faveur de l'intellect agent, car dans la génération d'un vivant, il y a en fait trois instances: entre l'agent universel premier (Dieu) et l'agent partiçulier qui apporte la détermination (la semence), il faut intercaler un agent universel second, principe universel de la vie, à savoir le mouvement du Ciel. De même, dans l'ordre de l'intellection, entre l'agent universel premier, qu'est l'Intellect divin, et l'image sensible, qui apporte la détermination, il faut intercaler un agent intermédiaire qui est l'intellect agent immanent à l'âme, principe de la connaissance intellectuelle.

Pierre Schwarz oppose alors à Durand l'opinion massive «de l'école commune de tous les philosophes» $^{52}$. Sa stratégie, comme celle de ses prédécesseurs, est de faire apparaître la né-

intellectus agens sit pars imaginis que est memoria. Quod oportet ponere sensum agentem, tum propter excessum potentie sensitive supra sensibile, tum propter excessum actus sentiendi quia causari non potest sufficienter ab obiecto sensibili.»

49 «Petrus autem a Palude in quarto scripto distinctione xlix negat sensum agentem.»

50 «Licet propter presentiam obiecti sensibilis potentie sensitive non oporteat ponere sensum agentem nec propter impressionem speciei quia eiusdem rationis est in medio et organo. Tamen oportet ponere et propter excessu potentie sensitive supra sensibile et propter excessu actus sentiendi qui causari non potest sufficienter ab obiecto sensibili.»

51 "Quia sicut intellectus excedit sensibilia ita et sensus licet non tantum. Sed non obstante excedentia sensus ad sensibilia nihilominus non propter hoc ponitur sensus agens. igitur nec ponitur intellectus agens.»

52 «Alia est opinio communis scole omnium philosophorum, quod necesse est ponere intellectum agentem.» 
gation de l'intellect agent comme une singularité, ce qui dans la mentalité scolastique suffit à l'invalider. Aussi fait-il ensuite feu de tout bois, puisque les quatre arguments qu'il avance en faveur de l'existence de l'intellect agent proviennent en fait de quatre traditions distinctes, pour ne pas dire hétéroclites.

À tout Seigneur, tout honneur. D'après Pierre Schwarz, qui se garde bien de donner quelque référence que ce soit, saint Thomas d'Aquin fonde l'existence de l'intellect agent sur la nécessité d'une puissance active capable de transformer en intelligible en acte l'intelligible en puissance qui est l'objet de l'intellect uni au corps. Le rôle irremplaçable de l'intellect agent est ainsi d'assurer, comme le dit Averroès, le passage de l'ordre de l'intelligible en puissance à l'ordre de l'intelligible en acte.

Une objection fait valoir que ce passage ne postule pas l'existence d'une puissance active réelle puisque, comme le faisaient déjà observer les partisans d'une pure abstraction de raison (Henri de Gand, Godefroid de Fontaines...), l'universel, qui est l'intelligible en acte, n'ajoute ni ne retranche rien de réel à l'image particulière. Pierre Schwarz répond longuement à cette objection. L'objet certes ne change pas en lui-même, mais un changement réel se produit dans l'intellect possible avec la production de la species intelligible, qui est universelle par représentation, dépouillée des conditions matérielles, à la fois en raison de son sujet (l'intellect possible) et de sa cause (l'intellect agent). Or, dans le cas de réalités relatives, il suffit qu'un seul des deux termes en relation connaisse un changement réel pour qu'on puisse légitimement parler d'un changement dans l'autre. Ainsi peut-on dire qu'avec la production de la species, l'objet «devient» universel.

Après saint Thomas, vient l'inévitable Hervé de Nédellec. Le principe qui le pousse à reconnaître l'existence de l'intellect agent est voisin de celui attribué à saint Thomas: l'objet matériel et l'intellect n'appartenant pas au même ordre de réalité, il faut rendre raison du passage d'un ordre à l'autre. Toutefois, Hervé insiste davantage sur la causalité productrice à l'œuvre dans la processus noétique. Il doit y avoir une proportion entre un agent et son effet. Aucune réalité matérielle ne peut donc agir par elle-même sur l'esprit. Il faut dès lors admettre une puissance active spirituelle et immatérielle qui «soit meut l'intellect possible par elle-même, soit en vertu de laquelle l'intellect possible est mû par l'objet. Nous l'appelons intellect agent ${ }^{53}{ }_{\text {». }}$.

Jean Duns Scot, qui d'ordinaire figure plutôt dans le camp des adversaires, devient ici un allié puisque Pierre Schwarz lui emprunte le troisième argument ${ }^{54}$. L'intellect possible ne peut intelliger qu'après que l'objet lui a été rendu présent sous mode d'objet, c'est-à-dire comme universel. Il faut donc que la quiddité matérielle qui est dans l'image soit rendue présente à l'intellect sur un mode universel avant que d'être intelligée. Cette universalisation ou abstraction ne peut être le fait d'une puissance sensible, ni de l'intellect possible puisque, répétons-le, l'universalité précède l'intellection, acte de l'intellect possible. Il faut donc admettre l'existence d'un intellect agent qui rend universelle la nature et la propose comme objet à l'intellect possible.

Enfin, Pierre Schwarz propose une argumentation qui semble de son crû: arguo sic. Partant à la recherche de la cause efficiente de l'intellection, il aboutit par élimination successive des candidats possibles à l'intellect agent. Il procède en deux étapes. Dans une première étape, un syllogisme écarte le candidat le plus sérieux, l'intellect possible. La majeure reprend le leitmotiv de Pierre Schwarz selon lequel il est impossible qu'une chose soit en même temps en puissance et en acte, qu'elle soit en même temps sujet et cause efficiente par rapport à un seul et même acte. La mineure rappelle alors ce que tous admettent, à savoir que l'intellect possi-

53 «Igitur oportet ponere aliquam virtutem spiritualem et immaterialem que vel per se solum moveat intellectum possibilem vel in cuius virtute intellectus possibilis ab obiecto moveatur. Sed tale vocamus intellectum agentem.»

54 Cf., par ex., JeAn Duns Scot, Ordinatio, I, dist. 3, p. 3, q. 1 (Opera omnia, III, Vatican, 1954, p. 209 ss.). 
ble est le sujet de l'intellection. Il ne reste plus qu'à conclure que l'intellect possible ne peut en aucun cas être la cause efficiente de l'intellection.

Dans la seconde étape, Pierre explique que la cause de l'intellection ne peut non plus être ni l'objet extra-mental (ipsa albedo) ni sa species sensible, car il y a disproportion entre la cause (matérielle) et l'effet (spirituel). Serait-ce alors la species intelligible qui existe subjectivement dans l'intellect possible? Mais, justement, quelle est la cause de cette species? Bref, devant la défection de tous les candidats, il ne reste donc qu'une solution: l'acte d'intelliger est causé par une cause $\mathrm{X}$ qu'on appelle l'intellect agent. L'intellect agent se définit donc nominalement comme ce qui cause l'acte d'intelliger.

Ces quatre arguments —objecte-t-on— prouvent bien la nécessité d'un intellect agent mais ils n'indiquent aucunement que celui-ci doive être immanent à l'âme, c'est-à-dire qu'il existe formellement en elle. Deux arguments vont donc le préciser. Le premier est strictement parallèle à la réponse faite à Durand qui estimait suffisante pour l'intellection l'existence d'un premier Intellect séparé. Le second, reprenant le grand principe de saint Thomas contre Averroès, fait valoir que nous expérimentons en nous-mêmes que nous sommes bien les sujets de l'acte de penser ${ }^{55}$. On le voit, la controverse dite «monopsychiste» ne subsiste qu'à l'état résiduel. Elle a apparemment perdu toute actualité pour Pierre Schwarz, qui est davantage soucieux de réfuter Durand qu'Averroès:

\section{L'ACTIVITÉ DE L'INTELLECT AGENT (Q. 50).}

La totalité de l'importante q. 50 (7 colonnes de l'édition de 1481, alors que la moyenne dans la section que nous considérons est de 5,4 colonnes) est consacrée à justifier et à préciser le rôle de l'intellect agent dans le processus noétique. Pierre Schwarz le fait toujours dans la perspective d'une réfutation de la théorie de Durand de Saint-Pourçain selon laquelle on peut expliquer l'intellection sans recourir à l'action de l'intellect agent.

Pierre a repéré trois «opinions». Il rattache la première à Durand et la résume en trois thèses et deux conséquences. La deuxième est celle d'Hervé de Nédellec et la troisième celle de Guillaume de Maricalm.

\section{A. Réfutation des positions attribuées à Durand}

Pierre attribue à Durand trois thèses (conclusiones) qu'il s'applique à réfuter. La première énonce que l'intellect agent n'agit pas sur l'intellect possible. Comment en effet l'âme pourrait-elle être à la fois en acte et en puissance? À quoi Pierre Schwarz répond qu'il n'y a là rien de contradictoire puisque l'âme possède des puissances qui sont essentiellement distinctes d'elle et distinctes entre elles. Quant à prétendre comme Durand que l'intellect agent en tant que cause de l'intellection devrait être connu en tout acte d'intellection à la manière dont la lumière est vue en tout acte de vision, on ne saurait l'accorder car la lumière est caùse par manière d'objet, pas l'intellect agent. On ne peut non plus prétendre que l'action causale universelle de l'intellect agent rendrait inutile l'action de la cause particulière (l'image). En effet, ces deux causes ne se situent pas dans le même ordre et ne se font donc pas concurrence.

La deuxième thèse que Pierre Schwarz prête à Durand (mais qui est loin d'être propre à ce dernier) nie que l'intellect agent imprime quelque forme que ce soit dans l'image. Si tel était le cas, nous dit-on, la species intelligible et l'acte d'intelliger porteraient la ressemblance de cette forme, alors qu'en fait ils portent la ressemblance de l'objet. Réponse de Pierre Schwarz:

55 «Quia omnis illius actionis naturalis quam experimur in nobis et perficitur ex conatu naturali, oportet ponere aliquod principium operationis in nobis effectivum ipsius.» 
le fils ne ressemble pas au soleil, qui est la cause universelle de sa génération, mais il ressemble à son père, qui est sa cause prochaine déterminante et spécificatrice. De même, la spécification de l'intellection vient de l'objet et non pas de l'intellect agent. Cette réponse suggère que le rapport entre l'image et l'intellect agent est celui de la cause seconde déterminante à la cause première universelle.

En outre, objectait-on, cette forme ou virtus communiquée par l'intellect agent à l'image sensible serait matérielle puisqu'elle serait reçue dans un sujet matériel. On voit mal alors en quoi la présence de cette virtus dans l'image permettrait de résoudre le problème de l'abstraction qui est celui du passage du matériel au spirituel. Pierre répond que cette vertu est matérielle quant à son sujet mais immatérielle quant à sa causalitée ${ }^{56}$. De même que l'eau qui ne peut réchauffer en vertu de sa nature propre réchauffe à titre d'instrument lorsque la forme de la chaleur est imprimée en elle par le feu, de même l'image produit instrumentalement un changement dans l'esprit lorsqu'elle est surélevée par la forme que lui communique l'intellect agent.

La troisième conclusion attribuée à Durand est que l'intellect possible n'exerce aucune action causale mais fortifie l'image par sa simple présence (praesentialis assistentia) et la rend capable d'agir sur l'intellect possible. Un peu comme les corps lourds, sans qu'aucun poids leur soit pourtant ajouté, gagnent en gravité lorsqu'ils se rapprochent du centre de la terre. On voit mal comment Pierre Schwarz a pu en venir à attribuer cette thèse de l'assistentia à Durand qui ne la mentionne ${ }^{57}$ que pour la critiquer sans ménagement:

«ll est étonnant qu'une chose donne à une autre un pouvoir et cependant n'influe pas sur elle ni ne supprime d'obstacle mais se contente d'être présente. En effet, cela semble impliquer contradiction: elle donne un pouvoir sans rien donner ${ }^{58}$ !»

Cette théorie semble plutôt correspondre à un enseignement d'Hervé de Nédellec ${ }^{59}$. Quoi qu'il en soit, Pierre Schwarz estime que cette théorie aboutit à la destruction pure et simple de l'intellect agent et il la réfute au moyen de quatre arguments. Il insiste fortement sur l'action réelle de l'intellect agent qui doit produire un effet réel, ce dont la théorie de l'assistentia ne permet pas de rendre compte.

\section{B. La théorie d'Hervé de Nédellec}

À la négation de l'intellect agent, Pierre Schwarz oppose deux théories «thomistes» qui rendent raison de son existence. La première, que notre auteur estime multum probabilis, c'està-dire fondée en raison, est celle qu'Hervé de Nédellec développe à la d. 17 du livre II de son commentaire des Sentences:

«L'intellect agent agit sur l'intellect possible en même temps que l'image sans qu'il imprime rien dans l'image, ni l'image en lui. Mais ils se comportent comme deux agents

56 Il dit s'inspirer d'Hervé. Cf. In II Sent, d. 17, q. 2, a. 3, ad 2 (p. 255b), où Hervé ne fait toutefois que rapporter de façon neutre la réponse de ceux qui tiennent l'existence de cette vertu imprimée dans l'image..

57 Cf. DurAND, op. cit. (f. $27 \mathrm{va}, \mathrm{n}^{\circ} 14$ ): «Sed diceret aliquis quod haec divisio sit insufficiens quia intellectus agens agit in phantasmata dando eis virtutem movendi intellectum possibilem non quidem aliquid imprimendo nec abstrahendo, sed solum assistendo et ponitur simile, quia lumen dat colori virtutem movendi visum, et tamen nihil imprimit colori nec a colore aliquid abstrahit sed tantum assistit, et eodem modo potest esse circa intellectum agentem et phantasmata ut videtur.»

$58 \mathrm{Ibid}$. (f. $27 \mathrm{va}, \mathrm{n}^{\circ} 15$ ): «Istud autem est mirabile quod aliquid det alteri virtutem, et tamen non influat, nec impedimentum tollat sed solum assistat. Hoc enim videtur implicare contradictionem, scilicet quod det virtutem nihil dando.»

59 En effet, la notion d'assistentia revient chez Durand au f. $28 \mathrm{ra}, \mathrm{n}^{\circ} 25$ à propos de la théorie d'Hervé: «Nec secundum hanc opinionem intellectus agens aliquid influit phantasmatibus, nec aliquid aufert ab eis sed solum coassistit.》 
imparfaits qui jouent le rôle d'un seul agent parfait, de sorte que l'un ne suffit pas sans l'autre ${ }^{60}$.»

Cette théorie d'Hervé est fort peu thomiste puisqu'elle reprend l'enseignement de Duns $S \cot ^{61}$ et met en cuvre la conception typiquement scotiste de la causalité concourante non réciproque de causes partielles ${ }^{62}$. Mais elle a eu une réelle postérité dans la tradition thomiste médiévale ${ }^{63}$. Durand l'avait sérieusement prise à partie, de sorte que Pierre Schwarz se croit tenu de justifier la position scotiste d'Hervé face aux objections de Durand ${ }^{64}$.

Primo, l'imperfection de l'action d'un agent provient soit d'une faiblesse de l'agent soit d'un empêchement résultant d'une action contraire, ce qui semble impossible dans le cas de l'intellect agent. Faux, réplique Pierre. L'intellect agent est parfait dans son ordre mais il n'est pas dans sa nature d'être l'agent total de l'intellection. De même, le soleil est parfait dans son ordre mais il ne peut engendrer un âne sans la collaboration d'un Brunellus (l'âne scolastique) quelconque qui engendre.

Secundo, l'effet ressemble à l'agent, affirme Durand. Par suite, si l'intellect agent était cause de l'intellection avec l'image, l'intellection devrait ressembler non seulement à l'image mais aussi à l'intellect agent. Pierre répond que la règle n'est pas universellement valable. Par exemple, l'effet d'une cause nécessaire peut être contingent. Elle ne vaut que lorsque les agents sont du même ordre.

Tertio, dans le cas de deux agents imparfaits, l'intensification de l'un en arrive à rendre l'autre inutile. Mais, réplique Pierre, cela ne vaut que lorsque les agents sont du même ordre, ce qui n'est pas le cas de l'image et de l'intellect agent. Il en va comme de l'homme et de la femme dans la génération. Mille femmes, dit-il, ne suffisent pas pour concevoir un enfant s'il n'y a pas d'homme! Comme on le voit, l'essentiel de l'apologie d'Hervé par Pierre Schwarz repose sur la distinction «qualitative» des deux causes concourantes: elles ne sont pas du même ordre.

\section{La théorie de Guillaume de Maricalm}

La troisième opinion avancée par Pierre Schwarz -et à laquelle il semble se rallier- est celle de Guillaume de Maricalm: «L'intellect agent imprime une vertu réelle dans l'image ${ }^{65}$.» Alors qu'Hervé de Nédellec n'évoquait une action directe de l'intellect agent sur l'image que pour la révoquer ${ }^{66}$ et prôner le modèle rival d'une action concourante de l'intellect agent et de

60 "Secunda opinio est herphei quod intellectus agens agit in intellectum possibilem una cum phantasmate absque hoc quod imprimat aliquid fantasmati aut fantasmata sibi, sed se habent sicut duo agentia imperfecta supplentia vicem unius agentis perfecti ita quod unum non sufficit sine altero.»

61 Cf., par exemple, JeAn Duns Scot, Ordinatio, I, d. 3, p. 3, q. 2 (Opera omnia, III, p. 293-295).

62 Sur cette notion et ses implications philosophiques, cf. A. DE MURALT, L'Enjeu de la philosophie médiévale, Etudes thomistes, scotistes, occamiennes et grégoriennes, «Studien und Texte zur Geistesgeschichte des Mittelalters, 24», Leiden, 1991, p. 32 ss. et p. 410. De fait, Jean Duns Scot explique la connaissance en recourant à ce schéma d'une causalité concourante non réciproque de l'intellect et de l'objet, qui sont donc des causes partielles, même si l'intellect est cause plus principale. Cf. par exemple, Lectura, I, d. 3, p. 3, q. 2-3.

63 Henri de Lubeck s'y rallie vers $1323 / 25$ dans son Quodlibet $I I, 5$, a. 3 (Cf. Ms Wien, Hofbibliothek, cod. Iat. $1382,177 \mathrm{rb}$-va.). Durandelle reconnaît le caractère fondé de cette théorie mais semble éprouver quelque doute sur son caractère thomiste, cf. Evidentiae contra Durandum, I, 4: «Aliud autem membrum, scilicet quod concurrant tanquam duo agentia, supplentia vicem unius perfecti agentis, non est de intentione Doctoris, eo modo saltem quo iste ponit. Nec istum modum, quanquam sit multum probabilis, intendo hic tenere, quia patet via facilior.»

64 Ces objections ne correspondent pas à celles que l'on trouve dans la rédaction C du Commentaire de Durand.

65 «Tertia opinio est magistri Guillielmi de Maricalmo quod intellectus agens imprimit realem virtutem in fantasma.»

66 Cf. In II Sent., d. 17, q. 2, a. 2 (p. 254a): «Licet autem istud posset aliquo modo sustentari, tamen difficile mihi videtur posse videre quid possit intellectus agens imprimere fantasmati.» 
l'image sur l'intellect possible, Guillaume de Maricalm affirme sans ambages que l'intellect agent agit directement sur l'image et indirectement, c'est-à-dire par la médiation instrumentale de l'image, sur l'intellect possible.

Deux arguments rendent ce modèle crédible. Primo, tout le monde accorde que l'image ne peut agir par elle-même sur l'intellect possible et que seule l'action de l'intellect agent la rend capable d'une telle action causale. Il faut donc que l'image reçoive de l'intellect agent «quelque vertu réelle». Un peu comme il faut recevoir la forme surajoutée de la charité pour agir surnaturellement.

Secundo, autant deux agents particuliers de même niveau peuvent agir sur un tiers sans agir l'un sur l'autre, autant c'est impossible pour le concours d'un agent universel et d'un agent particulier. Dans ce dernier cas, il faut soit que la cause universelle cause la cause particulière (mais l'intellect agent ne cause pas l'image) soit qu'elle imprime en elle une vertu active. Guillaume de Maricalm, en subordonnant la causalité de l'image à celle de l'intellect agent, prend nettement congé du modèle scotiste d'Hervé et, de fait, leurs explications du processus noétique sont incompatibles.

Au terme, Pierre explicite en quoi le modèle de maître Guillaume satisfait aux descriptions communes de l'intellect agent: il illumine, purifie et abstrait. L'intellect agent «illumine les images», en ce sens qu'il leur communique réellement une certaine lumière spirituelle. Un peu comme la nuit les chats, qui ont en eux une lumière, la communiquent à l'objet qu'ils rendent ainsi visible ${ }^{67}$. L'intellect agent «purifie les images». Il dissipe une certaine obscurité présente dans l'image (entendons, probablement, une certaine potentialité qu'il diminue en irradiant l'image de son actualité) mais surtout il produit avec l'image une species intelligible, forme qui représente l'objet de façon universelle, «purifiée» des conditions matérielles individuantes. Enfin l'intellect agent «abstrait». Là encore, cette abstraction doit s'entendre du côté de l'effet, c'est-à-dire de la species intelligible. Elle est en effet «tirée» de l'image vers l'intellect. Elle représente en faisant abstraction des conditions individuantes de sorte que «ce qui appartient à la species en réalité appartient à l'objet représenté par elle selon la raison et non selon la réalité68).

Quatre problèmes (dubia) qui semblent militer contre la solution de Guillaume de Maricalm permettent en fait d'affiner son modèle noétique. Une première difficulté se rencontre déjà chez Durand: il est généralement admis qu'un ange, substance immatérielle créée, ne peut imprimer directement une forme dans la matière ${ }^{69}$. A plus forte raison, l'intellect agent ne peutil rien imprimer dans l'image matérielle ${ }^{70}$. Guillaume de Maricalm (iste doctor) répond en distinguant le cas de l'ange et celui de l'intellect agent. Si l'ange, dit-il, n'imprime pas de forme dans la matière, c'est parce qu'il existe d'autres agents dont c'est la fonction propre (le ciel...). Réponse un peu courte, avouons-le, qui confond le fait avec le droit. Par contre, poursuit Guillaume, les images ne peuvent mouvoir par elles-mêmes l'intellect possible et il faut donc admettre l'existence d'un principe qui les en rende capables.

67. On objectera plus loin (dubium 3) que cette lumière n'agit que sur le milieu et non pas sur l'objet. À quoi Pierre Schwarz répondra que l'objet de la vue doit s'entendre au sens large: il désigne l'ensemble formé et par l'objet visible et par le milieu.

68 «Realiter representat sine conditionibus individuantibus et quod speciei competit secundum rem competit obiecto representato per ipsam secundum rationem et non secundum rem.»

69 Cf., par exemple, THomas D'AQUIN, Summa theologiae Ia, q. 105, a. 1.

70 Cf. DURAND, op. cit. (f. $27 \mathrm{rb}, \mathrm{n}^{\circ}$ 6); «Secundo, quia si Angelus non potest in materia corporali imprimere formam immediate, videtur quod multo minus hoc posset intellectus agens, phantasmata autem est quid corporeum, ergo intellectus agens nullam formam potest phantasmatibus imprimere.» 
La deuxième objection, classique elle aussi, qui apparaît chez Durand ${ }^{71}$, mais se trouvait déjà chez Godefroid de Fontaines ${ }^{72}$, Hervé de Nédellec ${ }^{73}$ et tant d'autres, fait valoir que la vertu que l'intellect agent est censé imprimer dans l'image ne peut qu'épouser la condition ontologique du sujet récepteur. Elle sera donc matérielle et particulière comme l'image, de sorte qu'elle ne permet pas plus d'agir sur l'intellect possible que l'image seule. Certains, répond Guillaume de Maricalm cité par Pierre Schwarz, pensent là contre que cette vertu est effectivement spirituelle et qu'elle est dans l'image un peu à la manière dont l'âme est dans le corps, mais Guillaume rétorque qu'effectivement une virtus purement spirituelle ne peut exister dans un corps. Il faut plutôt tenir que «cette virtus imprimée [dans l'image], bien qu'elle soit matérielle subiective est cependant spirituelle ou immatérielle de façon virtuelle et du point de vue de la causalité efficiente ${ }^{74}$ ».

Mais cette virtus impressa ne fait-elle pas concurrence à la species intelligible, se demande une quatrième objection? C'est en effet la. species qui fait de la réalité singulière présente dans l'imagination un objet universel pour l'intellect. Guillaume répond qu'il y a équivoque sur le terme d'objet. L'objet est soit la cause motrice d'une puissance soit le terme atteint par son action. L'objet qui meut l'intellect possible est l'image en vertu de cette virtus impressa. Cet objet précède logiquement la species dont il est le principe. Mais l'objet universel, présent à l'intellect, auquel se termine l'action est la species intelligible.

\section{INTELLECT AGENT ET INTELLECT POSSIBLE (Q. 51)}

La q. 51 traite de la distinction entre l'intellect agent et l'intellect possible. Par delà les q. 49-50, elle se situe dans le prolongement de la q. 48 sur la distinction entre l'âme et ses puissances, à laquelle Pierre Schwarz renvoie d'ailleurs de façon explicite.

Dans cette longue q. 48 (10 colonnes) — les puissances de l'âme se distinguent-elles de l'âme?»—, Pierre Schwarz, en s'inspirant surtout de Jean Cabrol ${ }^{75}$, avait établi contre Jean Duns Scot, puis contre Guillaume d'Ockham, la thèse effectivement thomiste de la distinction réelle et essentielle entre l'âme et ses diverses puissances. Cette thèse générale constitue le cadre dans lequel il peut affirmer la distinction de l'intellect agent et de l'intellect possible.

Au début de la q. 51, notre dominicain annonce trois opinions. En fait, il n'en expose que deux, pour les réfuter, celle d'Ockham et celle de Scot, la troisième s'en déduisant de quelque manière. L'opinion d'Ockham, peu favorable à la multiplication des entités, affirme l'identité réelle de l'intellect agent et de l'intellect possible ${ }^{76}$. Elle repose sur le principe fort simple de la transitivité: puisque les puissances de l'âme sont réellement identiques à l'âme, elles doivent aussi être réellement identiques entre elles. Pierre Schwarz lui oppose que les propriétés réelles contradictoires qui sont celles de l'intellect agent et de l'intellect possible — «L'intellect

71 Cf. ibid., $\mathrm{n}^{\circ} 5$

72 Godefrom de FonTAINEs, Quodlibet V, q. 10 (ed. de Wulf, J. Hoffmans, Louvain, 1914, p. 36): «Quod enim intellectus agens primo et immediate agat aliquid sive aliquam dispositionem vel virtutem circa phantasmata ipsa, qua phantasmata quae prius erant potentia intelligibilia fiant actu intelligibilia sive in actu moventia intellectum possibilem, non videtur. Nam quicquid fieri posset in phantasmatibus quae sunt in phantasia esset aliquid in phantasia organica et haberet esse signatum sicut ipsa phantasmata, et per huiusmodi dispositionem non possent fieri phantasmata intelligibilia»; p. 37: «Omnis dispositio possibilis esse in phantasmate vel in phantastico est singulare et modum singularis habens, cum tali autem dispositione non potest phantasmata movere intellectum.»

73 Cf. In II Sent., d. 17, a. 3 (p. 253b-254a).

74 «Vult igitur iste doctor quod ista virtus impressa licet sit materialis subiective est tamen spiritualis sive immaterialis virtualiter et effective.»

75 Cf. Jean Cabrol, Defensiones, I, d. 3, a. 2 (t. 1, p. 189 ss).

76 «Intellectus agens et possibilis sunt omnino una res et cum essentia anime et inter se et differunt solum secundum rationem.» 
agent cause réellement l'intellection et ne la reçoit pas. L'intellect possible reçoit réellement l'intellection et ne la cause pas ${ }^{77} \gg$ - ne peuvent coexister dans un seul et même sujet.

La deuxième opinion est celle de Jean Duns Scot:

«L'intellect agent et l'intellect possible sont une seule réalité absolue et ne peuvent différer comme deux réalités absolues, mais ils diffèrent en fonction d'une diversité de points de vue ${ }^{78} . \gg$

Bref, l'intellect agent, c'est l'intellect considéré comme actif, et l'intellect possible, l'intellect considéré comme passif. Pierre Schwarz avance alors cinq arguments scotistes, dont je ne peux préciser la provenance exacte, auxquels il s'applique à répondre.

Les quatre premiers ont en commun de supposer que, si l'intellect agent était réellement distinct de l'intellect possible, il y aurait cette contradiction que l'intellect agent serait à la fois plus noble et moins noble que l'intellect possible. D'une part, en effet, l'agent vaut toujours mieux que le patient, mais, d'autre part, l'intellect possible possède certaines propriétés qui semblent le mettre au-dessus de l'intellect agent. Primo, il possède la perfection de la connaissance alors que l'intellect agent en est dépourvu. Secundo, il est plus proche que l'intellect agent de la fin qu'est l'acte d'intelliger: il en est le sujet récepteur, alors que l'intellect agent ne l'atteint que par la médiation de l'image. Tertio, il demeure dans la vision béatifique, alors que l'intellect agent n'y exerce plus aucune activité. Quarto, il est un élément constitutif de l'image de la Trinité en l'homme, ce qui n'est pas le cas de l'intellect agent, lequel ne pourrait relever que de la mémoire, mais, justement, il ne conserve pas les species.

À chacun de ces arguments, Pierre répond qu'il se retourne en fait contre Scot. Mais il les réfute aussi directement. Primo, l'intellect agent ne connaît certes pas mais il fait connaître. Il est donc plus noble que l'intellect possible qui ne fait que recevoir la connaissance. Secundo, Pierre conteste la valeur universelle du principe mis en cuvre et nie que ce qui est plus proche de la fin dans l'ordre de la cause matérielle (l'intellect possible) vaille mieux que ce qui en est moins proche dans l'ordre de l'efficience (l'intellect agent). Le mouvement de la main qui verse la potion au malade est plus proche de la fin qu'est la santé que l'art du médecin et pourtant celui-ci est plus parfait. Tertio, l'intellect possible ne cause pas la vision béatifique au plan de l'efficience, il la reçoit. Or —réponse à faire frémir H. De Lubac!一, il vaut mieux, estime Pierre, être un principe actif dans l'ordre naturel qu'un principe passif dans l'ordre surnaturel, un peu comme il vaut mieux être principe actif dans la production d'un cheval que simple principe passif dans la production de l'âme rationnelle ${ }^{79}$. Quarto, l'intellect agent appartient bel et bien à cette partie de l'image de la Trinité dans l'esprit qu'est la mémoire, non pas en tant que celle-ci reçoit les species mais en tant qu'elle engendre la connaissance.

Un cinquième argument de Scot fait valoir qu'il n'y a pas de contradiction à ce qu'un même sujet soit à la fois actif et passif. Pierre Schwarz renvoie alors à ses inusables développements sur l'impossibilité pour un sujet d'être à la fois en acte et en puissance, sur la non coïncidence de la cause matérielle et de la cause efficiente ${ }^{80}$.

77 «Intellectus agens realiter causat intellectionem et non suscipit eam. Intellectus autem possibilis realiter suscipit intellectionem et non causat eam.»

78 "Opinio est Scoti quod intellectus agens et possibilis sunt una res absoluta nec possunt differe sicut due res absolute, sed differunt secundum diversos respectus.»

79 «Perfectius est se habere active ad actum naturalem quam se habere passive ad actum supernaturalem.»

80 Pierre Schwarz rattache l'argument de Scot à ses positions sur le rapport acte-puissance: «Istam rationem habes deductam super Porphirio questione quinta conditione quinta subiecti scibilis dubio secundo et adducitur pro ratione Scoti.» Il renvoie alors à sa réfutation: «Contra istam opinionem adduc illas quinque rationes que adducte fuerunt in predicta questione iam [non?] nominata contra opinionem Scoti, ubi probatur quod impossibile est quod idem absolutum sit activum et passivum respectu eiusdem. Nec oportet aliquid mutare nisi terminos aliquos, scilicet ubi habetur subiectum et propria passio ponitur hic intellectus agens et possibilis.» Et plus loin: «Ad quintam dicendum sicut dictum est supra Porphirio questione quinta conditione subiecti scibilis dubio secundo ubi ponitur ista ratio in forma et eiusdem solutio.» 


\section{INTELLECT AGENT, SPECIES, MÉMOIRE (Q. 52-53)}

Autant la q. 51 révèle des positions résolument anti-scotistes, autant les deux questions suivantes, consacrées à la species intelligible, témoignent de l'imprégnation scotiste du thomisme de Pierre Schwarz. L'action de l'intellect agent étant immédiatement ordonnée à la production de la species intelligible, il était logique que Pierre s'intéresse de plus près à cette instance noétique qui ne manquait pas d'être contestée.

La q. 52 - «Est-il nécessaire de poser dans la partie intellective quelque species intelligible, outre celle qui est dans l'image?»— est de fait directement dirigée contre la thèse classique d'Henri de Gand ( $\dagger 1293$ ) selon lequel il n'existe pas de species intellectuelle ${ }^{81}$. Pour Henri, l'intellect va chercher ses informations dans des «species impresses», terme équivoque qui désigne, en fait, dans son vocabulaire, les images présentes dans la faculté sensible. Ces images sont de soi individuelles puisque matérielles, mais l'intellect agent a pour rôle de faire apparaitre en elles l'élément universel. Il ne s'agit donc pas d'une séparation réelle entre le contenu universel et les conditions individuantes, qui constituerait l'universel dans un nouvel état ontologique, mais d'une séparation virtuelle. L'intellect agent fait apparaître l'universel dans ce singulier qu'est l'image. L'image singulière et la species sont donc réellement identiques. Bref, comme le résume Pierre Schwarz, «il ne faut poser aucune species dans la partie intellective [de l'âme] mais celle qui est dans la partie sensitive suffit ${ }^{82}{ }^{\text {}}$.

L'exposé et la réfutation des arguments henriciens sont largement inspirés de Jean Duns Scot, dont on sait qu'il n'a rien passé au maître gantois ${ }^{83}$. Ainsi les cinq arguments contre la species intelligible se trouvent tous, et dans cet ordre, dans le texte même de Duns Scot. Quant aux réponses, elles résument sans recul celles données par Scot. Par exemple, la première objection faisait valoir que la species impresse représente l'objet sous l'angle où il cause cette impression. Or l'objet agit en tant que réalité singulière. La species produite représente donc l'objet comme singulier et non comme universel. Dans sa réponse, Pierre Schwarz procède, absolument comme Duns Scot, en deux étapes. Il propose d'abord de distinguer la ratio agendi (la nature) et la ratio agentis (le sujet individuel). Mais, comme Scot, il préfère une autre solution: l'objet n'est pas la cause totale de la species, mais il l'engendre avec le concours de l'intellect agent qui confère précisément à la species intelligible son statut d'universel.

Quant à la seconde opinion - « l'opinion commune de toute l'école des philosophes qui suivent Aristote au livre III $D e l^{\prime} a m^{84}{ }^{8}$, à laquelle Henri de Gand oppose sa singularité-, elle est présentée dans les termes mêmes du Docteur subtil. Pour prouver la nécessité d'une species intelligible, Pierre fait en effet valoir que l'universel doit être présent à l'intellect possible àvant même que celui-ci n'intellige, ce qui n'est possible que par la présence d'une species intelligible qui représente l'objet sous son aspect d'universalité. De l'aveu même de Pierre,

81 Sur la noétique d'Henri de Gand, cf., S. P. Maronne, Truth and Scientific Knowledge in the Thought of Henry of Gent, Cambrige (USA), 1985; K. H. TACHAU, Vision and Certitude in the Age of Ockham, Optics, Epistemology and the Foundations of Semantics 1250-1345, «Studien und Texte zur Geistesgeschichte des Mittelsalters, 22», Leiden, 1988, p. 28-39; A. DE LIBERA, La querelle des universaux, De Platon à la fin du Moyen Age, Paris, 1996, p. 307-313: «Les deux virages d'Henri de Gand»; L. SPRuIr, Species intelligibilis..., p. 205-212.

82 «Prima opinio Henrici de Gandavo quod in parte intellectiva nulla est ponenda species sed sufficit illa que est in parte sensitiva, scilicet in fantasmate.»

83 Cf. Ordinatio, I, dist. 3, pars 3, q. 1 (Opera omnia III, p. 201 ss et 231 ss); Lectura, I, dist. 3, pars 3, q. 1 (Opera omnia XVI, p. 325 ss et 341 ss). L'exposé des thèses d'Henri était un lieu commun, mais je ne vois pas que la présentation de Jean Cabrol, Defensiones, I, d. 35, q. 1 (t. 2, p. 353a-354a) ou celle du De quattuor materiis d'Hervé de Nédellec (P. Stella, «La prima critica di Hervaeus Natalis o.p. al noetica de Enrico di Gand: il 'De intellectu et specie' del cosidetto 'De quattuor materiis'», Salesianum 21 [1959], p. 125-170) aient exercé une influence littéraire directe sur notre question.

84 «Alia est opinio communis schole philosophorum sequentium Aristotelem in $3^{\circ}$ de anima qui dicit quod anima intellectiva est locus specierum intelligibilium.» 
le premier des cinq arguments en faveur de l'existence de la species intelligible, provient d'un bon scotiste (bonus scotus). En fait, ce sont les trois premiers arguments qui résument les développements par lesquels Scot démontre la nécessité de la species à partir de l'universalité (exigée) de l'objet ${ }^{85}$. L'objet formel de l'intellect est l'universel comme tel, lequel doit être présent à l'intellect avant qu'il n'intellige. Or seule la species qui est dans l'intellect peut représenter l'objet de façon universelle. Celle qui est dans le sens ne représente l'objet que sur un mode individuel. Il ne peut donc s'agir de la même species. Quant aux deux derniers arguments, ils reprennent ceux que Duns Scot développe pour démontrer la nécessité de la species à partir de la présence (exigée) de l'objet ${ }^{86}$.

$\mathrm{Au}$ milieu de ce résumé, Pierre Schwarz insère toutefois un dubium. Comment, dès lors, l'existence de la species intelligible ne rend-elle pas vaine la conversio ad phantasmata ${ }^{87}$ ? Si l'image n'est qu'un cocon provisoire, dont est extraite une species qui mène ensuite sa vie propre, comment justifier la place permanente que l'aristotélisme reconnaît à l'image dans la pensée? Pierre Schwarz rend raison du retour aux images de deux manières. Il se réfère d'abord au «saint docteur», c'est-à-dire à saint Thomas d'Aquin, dont il présente la position sur un mode nettement scotiste. La species étant causée à la fois par l'intellect agent et la species sensible comme «cause moins principale», elle porte la trace de son origine: «La species qui est dans l'intellect comme représentation porte la marque (caracterisatur) de l'image comme de sa cause, de sorte qu'elle ne représente rien si ce n'est selon la conversion à l'image même ${ }^{88}$ ». La seconde justification, anonyme (alii), prétend que la species intelligible est trop subtile pour agir sur une âme qui habite le corps: elle a besoin de la force de l'image à laquelle elle est liée, un peu comme la species de la blancheur qui est dans le milieu ne peut mouvoir le sens que moyennant la coexistence de la blancheur sur la surface du mur.

S'il n'y a de species que celle qui se trouve dans la partie sensitive de l'âme, comme le pense Henri de Gand, l'idée d'une mémoire proprement intellectuelle semble exclue. C'est pourquoi Pierre Schwarz, dans le prolongement de la critique de la noétique henricienne, traite à la q. 53 du problème suivant: «La mémoire est-elle dans la partie intellective ou bien dans la seule partie sensitive?» Pierre Schwarz écarte une première opinion qui récuse toute mémoire intellectuelle sous prétexte que le passé comme tel n'est pas intelligible et une deuxième qui écarte toute mémoire sensible sous prétexte que le temps ne peut être appréhendé que par l'intelligence. Il tient quant à lui, en s'appuyant essentiellement sur des arguments d'autorité, qu'il faut admettre et une mémoire sensible contenant des species sensibles et une mémoire intellectuelle contenant des species intelligibles.

Au terme, il soulève un dubium sur les rapports entre la mémoire (intellectuelle) et l'intelligence dont la réponse met bien en évidence les traits caractéristiques de la noétique qu'il adopte. Pierre distingue en effet trois cas:

- Si la mémoire désigne «le principe qui reçoit la species intelligible» et si l'intelligence désigne «le principe qui reçoit l'intellection formelle», alors mémoire et intelligence sont réellement identiques. Même si Pierre parle d'actes divers, c'est un seul et même principe qui reçoit et la species et la connaissance.

- Si la mémoire désigne «le principe producteur de la connaissance engendrée» et si l'intelligence désigne «le principe récepteur de la connaissance actuelle», alors mémoire et intelligence sont deux facultés réellement distinctes, car —une fois de plus - la cause efficiente et la cause matérielle ne peuvent jamais coïncider.

85 Cf. Ordinatio, I, d. 3, p. 3, q. 1 (p. 211 ss). Le premier argument de Pierre correspond aux n ${ }^{\circ} 352-356$; le deuxième aux $\mathrm{n}^{\circ} 357$; le troisième au $\mathrm{n}^{\circ} 359$.

86 Cf. Ibid. (p. 222 ss.). Le quatrième argument de Pierre correspond au $n^{\circ} 366$; le cinquième au $n^{\circ} 367$.

87 Jean Duns Scot rapportait déjà cette objection d'Henri de Gand, cf. Ordinatio, 1, d. 3, p. 3, q. 1 (p. 204 ).

88 «Ideo species que est in intellectu in representatione caracterisatur a fantasmate tanquam a sua causa ut nihil representet nisi secundum conversionem ad ipsum fantasma.» 
— Si la mémoire désigne «le principe actif de la connaissance engendrée» et l'intelligence «la connaissance formelle», la mémoire se distingue réellement de l'intelligence comme le tout se distingue réellement de ses parties. La mémoire ainsi entendue inclut en effet l'intellect agent, la species intelligible et l'acte d'intelliger.

Au terme de ce parcours analytique à travers un texte ingrat, il convient de tirer trois conclusions plus générales.

La première regarde l'histoire du thomisme. L'étude des questions relatives à la noétique dans le Clipeus thomistarum et spécialement la mise au jour, qui est pourtant loin d'être exhaustive, de leurs sources, interdit définitivement de considérer Pierre Schwarz comme un «grand» thomiste ni même comme un penseur de réelle envergure. Non seulement, selon la mode du temps, il pille sans originalité ses devanciers - généralement en les mentionnant-, mais, malgré quelques convictions fortes qui structurent son discours (par exemple, sur la nécessaire distinction entre le sujet récepteur d'une perfection et sa cause productrice), il peine à proposer une vision synthétique des problèmes et n'aboutit qu'à une forme d'éclectisme. Par exemple, sur la question du rôle respectif de l'intellect agent et des images dans la production de la species, il ne choisit pas franchement entre les modèles pourtant incompossibles d'Hervé de Nédellec, qui tient une causalité concourante, et de Guillaume de Maricalm, qui tient une subordination de l'image à l'action de l'intellect.

Cela dit, l'intérêt historique du Clipeus reste considérable. Primo, il illustre à merveilles la figure que peut prendre le thomisme dans un univers scolastique désormais figé: un thomisme scolaire, simplifié à outrance, et qui se définit largement par opposition aux autres écoles $^{89}$. Secundo, le Clipeus thomistarum témoigne d'un thomisme qui a largement coupé les ponts avec saint Thomas lui-même. Non seulement Pierre Schwarz a ingurgité une forte dose de scotisme, soit directement soit par l'intermédiaire d'Hervé de Nédellec, dont la conception de l'intellection doit beaucoup à son adversaire franciscain, non seulement il cite peu les textes mêmes de saint Thomas (et donc, probablement, les lit peu), mais il l'éclipse par ses disciples supposés. En effet, Pierre Schwarz cite plus longuement Hervé de Nédellec, Jean Cabrol ou Guillaume de Maricalm que l'Aquinate lui-même, dont les principales citations sont d'ailleurs souvent extraites des Defensiones de Cabrol! Bien plus, au plan doctrinal, Pierre Schwarz est manifestement plus hervéiste que thomiste ${ }^{90}$. La référence à une tradition d'interprétation est certes légitime, mais ici la tradition en arrive à ensabler sa propre source. Toutefois cette dérive n'est pas générale dans le thomisme du XV siècle. Les grands thomistes de l'époque (Jean Cabrol, Dominique de Flandres...) ont au contraire prôné une vive attention aux ipsissima verba de l'Aquinate.

La deuxième conclusion concerne l'histoire du thème de l'intellect agent. Les questions que le Clipeus lui consacre attestent que, depuis saint Thomas, l'intérêt s'est considérablement déplacé. La question du statut ontologique de l'intellect agent, si épineuse au XIII ${ }^{e}$ siècle dans le contexte de l'aristotélisme radical, a manifestement perdue de son actualité. Désormais, suite à la contestation durandienne, la question centrale est celle de la nécessité et de l'existence même d'un intellect agent. Elle est inséparable d'une réflexion systématique sur les processus de l'intellection et sur le rôle respectif qu'y jouent l'objet et les diverses instances cognitives.

Notre troisième conclusion porte sur l'orientation générale de la noétique hervéiste de Pierre Schwarz - pour autant qu'on puisse la reconstituer. Elle manifeste un souci louable de met-

90 En I, q. 32, Pierre ne peut pas ne pas mentionner l'opposition d'Hervé et de Thomas sur la question des rapports de l'être et de l'essence dans les créatures, mais tout en se ralliant à la position de saint Thomas, exposée par Jean Cabrol, il se garde bien de critiquer la position d'Hervé. 
tre en valeur la causalité de l'objet dans le processus noétique. Mais cela conduit malencontreusement à hypertrophier la passivité de l'intellect face à l'objet. Pierre Schwarz exténue en effet la vitalité propre de l'intellect possible. L'intellection n'est plus, comme chez saint Thomas, un acte vital de l'intellect possible préalablement fécondé et actualisé par l'objet sous l'influx de l'intellect agent. D' une part, le rapport entre la réception de la species intelligible produite par l'image et l'intellect agent et l'acte d'intellection n'est pas clair, car, même s'il distingue parfois la species intelligible de l'acte cognitif, Pierre Schwarz tend de manière générale à identifier l'intellection à la pure et simple réception passive de la species intelligible par l'intellect possible. Cet oubli de la spontanéité de l'esprit se traduit aussi par le silence assourdissant de Pierre Schwarz sur le verbe mental, fruit immanent de l'intellection, dont il ne souffle mot. D'autre part, Pierre Schwarz analyse le processus noétique sous l'angle privilégié de l'efficience productrice, au risque de réduire la pensée à une mécanique et de manquer la spécificité de la vie de l'esprit.

P. Serge-Thomas Bonino, O.P. Couvent Saint-Thomas d'Aquin

1, Impasse Lacordaire 31078 Toulouse Cedex 4

Francia 